\title{
A Single-Input Dual-Output Step-Up/Step-Down DC-DC Converter Designed by Switched Capacitor Techniques
}

\author{
Kei Eguchi ${ }^{\text {a,* }}$, Kanji Abe ${ }^{\mathrm{a}}$, Shinya Terada ${ }^{\mathrm{b}}$, Ichirou Oota ${ }^{\mathrm{b}}$ \\ ${ }^{\text {a }}$ Fukuoka Institute of Technology, 3-30-1 Wajirohigashi, Higashi-ku, Fukuoka, 811-0295 Japan \\ ${ }^{b}$ Kumamoto National College of Technology, 2659-2 Suya, Koushi, Kumamoto, 861-1102 Japan \\ *Corresponding Author: eguti@fit.ac.jp
}

\begin{abstract}
In this paper, we propose a single-input dual-output (SIDO) DC-DC converter designed by switched capacitor (SC) techniques. Unlike conventional SC converters, the proposed converter can provide nine kinds of two steppedup and/or stepped-down output voltages without changing circuit topology. This paper also presents a novel analysis method to estimate properties of the SC SIDO converters, because few studies have been done on the theoretical analysis of the multi-output SC converters. Unlike the traditional state-space averaging method, the proposed method can derive the power efficiency and output voltages without complex matrix calculations. The simulation program with integrated circuit emphasis (SPICE) simulation shows the following results: (1) more than $82 \%$ efficiency is obtained over a range of output power from $0.1 \mathrm{~W}$ to $1 \mathrm{~W}$ with conversion ratios of $1 / 2$ and $3 / 2$ and (2) the proposed analysis method will be helpful to estimate the power efficiency and output voltages of the SC SIDO converter, because theoretical results are in good agreement with SPICE simulated results. Furthermore, experiment on a breadboard shows the validity of the proposed SC SIDO topology.
\end{abstract}

Keywords: switched capacitor circuits, DC-DC converters, single-input dual-output, step-up/step-down converters.

\section{Introduction}

In mobile consumer products, power supplies at different voltage and/or current levels are required to drive some circuit units. To generate multiple outputs at different voltage levels from a single DC supply, several DC power distributed systems have been used: low dropped regulator
(LDO) arrays, single-input parallel-connected DC-DC converters, and so on. Among others, in order to reduce the number of external circuit components, single-inductor multi-output (SIMO) DC-DC converters have been developed in recent years. For example, Patra et al. and Deepti et al. suggested a step-down SIMO converter using a buck converter topology ${ }^{(1,2)}$. Owing to the single inductor topology, the step-down SIMO converter ${ }^{(1,2)}$ can achieve small hardware cost. However, the converter reported in references $(1,2)$ offers only stepped-down voltages. Ray et al. proposed a multi-output DC-DC converter ${ }^{(3,4)}$ which can provide a step-up and multiple step-down outputs. Unlike the step-down SIMO converter ${ }^{(1,2)}$, the multi-output converter reported in references $(3,4)$ can provide stepped-up and stepped-down voltages. However, the multioutput converter reported in references $(3,4)$ requires a couple of inductors. To drive multi-color LEDs, Dietrich et al. suggested an SIMO converter using a buck-boost converter topology ${ }^{(5)}$. Unlike the conventional converters reported in references (1-4), the buck-boost SIMO converter $^{(5)}$ can achieve step-up and step-down conversion by using only one inductor. However, the outputs are negative voltages though the buck-boost SIMO converter ${ }^{(5)}$ can drive multi-color LEDs effectively. Therefore, the applications field of the buck-boost SIMO converter is limited.

As distinct from above-mentioned DC-DC converters containing magnetic components, multi-output DC-DC converters designed by switched-capacitor (SC) techniques have been proposed recently. The SC DC-DC converter can be realized without the use of magnetic components. Therefore, the SC converter can reduce not only circuit size but also effects of the electromagnetic interference (EMI), though the conversion ratio of the SC converter is 
predetermined by circuit topology. In previous studies, Suzuki et al. proposed a serial fix type DC-DC converter ${ }^{(6)}$ designed by SC techniques. However, the serial fix type converter provides only the stepped-down voltages, $V_{i n} / N$ $(N=1,2,3, \ldots)$. Of course, as reported in the reference [7], the conventional converter can generate stepped-up voltages by swapping the input and output terminals. However, the serial fix type converter cannot control conversion ratios without changing circuit topology. Hua et al. suggested a single-input dual-output (SIDO) DC-DC converter for energy harvesting applications ${ }^{(8)}$. By using a charge pump ${ }^{(9)}$, the SIDO converter reported in reference (8) can provide stepped-up voltages from a small input voltage. However, the conversion ratios of the conventional SIDO converter reported in reference (8) are only $2 \mathrm{x}$ and $3 \mathrm{x}$. Chen et al. proposed an SC SIDO converter employing pseudo-three phase swap-and-cross control $^{(10)}$. By connecting series-parallel type converters ${ }^{(11)}$ in parallel, the conventional converter reported in reference (10) achieves step-up and step-down conversion. However, the circuit size becomes large, because two converters are required to achieve step-up/step-down conversion.

In this paper, we propose an SIDO step-up/step-down converter designed by SC techniques. The proposed SIDO converter consists of 15 transistor switches and 3 capacitors. Unlike conventional SC converters ${ }^{(6-11)}$, the proposed converter can offer nine kinds of two stepped-up/ stepped-down outputs: $V_{i n} / 3$ and $2 V_{i n} / 3, V_{i n} / 3$ and $V_{i n}, 2 V_{i n} / 3$ and $V_{i n}, V_{i n} / 2$ and $V_{i n}, V_{i n}$ and $3 V_{i n} / 2, V_{i n} / 2$ and $3 V_{i n} / 2, V_{i n}$ and $2 V_{i n}, V_{i n}$ and $3 V_{i n}$, and $2 V_{i n}$ and $3 V_{i n}$. This paper also presents a novel analysis method to estimate properties of the SC SIDO converters, because few studies have been done on the theoretical analysis of the SC SIDO converters. In the traditional theoretical analysis of SC DC-DC converters, the state-space averaging method has been commonly used ${ }^{(12,13)}$. However, the state-space averaging method requires complex matrix calculations. By assuming a five-terminal equivalent circuit, the proposed method derives the power efficiency and output voltages without complex matrix calculations. To confirm the validity of the proposed converter, simulation program with integrated circuit emphasis (SPICE) simulations, theoretical analysis, and experiments are performed.

The rest of this paper is organized as follows. In Section 2 , the circuit configuration of the conventional SC SIDO converter and the proposed SC SIDO converter is presented. In Section 3, the property of the proposed converter is analyzed by the proposed analysis method. Simulation results and experimental results are shown in Sections 4 and 5 , respectively. Finally, conclusion and future work are drawn in Section 6.

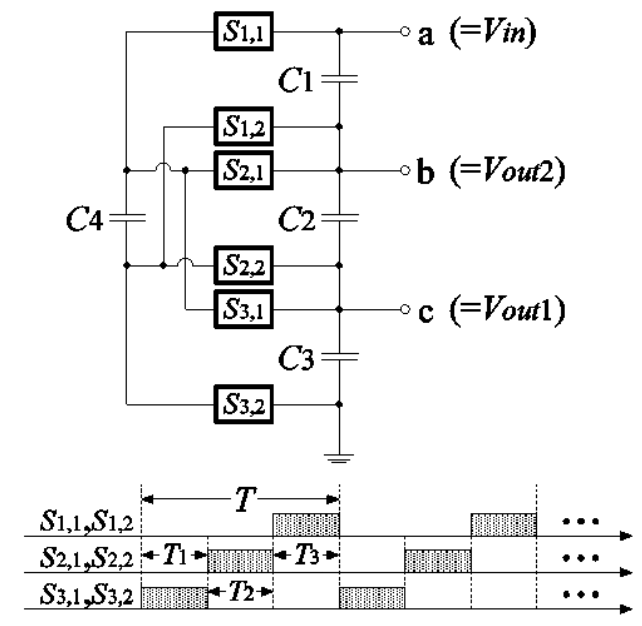

Fig. 1. Conventional dual-output SC DC-DC converter.

\section{Circuit Configuration}

\subsection{Conventional Converter}

Figure 1 shows an example of the conventional multi-output SC converter. The converter of figure 1 is based on the serial fix type converter ${ }^{(6)}$ proposed by Suzuki et al. The conventional converter consists of six transistor switches and four capacitors, where transistor switches $S_{1, j}$, $S_{2, j}$, and $S_{3, j}(j=1,2)$ are driven by non-overlapped three phase clock pulses. By controlling theses transistor switches, the conventional converter of figure 1 offers the following stepped-down voltages:

$$
V_{\text {out } 1}=\left(\frac{1}{3}\right) V_{\text {in }} \quad \text { and } \quad V_{\text {out } 2}=\left(\frac{2}{3}\right) V_{\text {in }} .
$$

In the conventional converter, a fly-capacitor $C_{4}$ shuttles electric charge among the three voltage regions: $V_{i n} / 3$, $2 V_{i n} / 3$ and $V_{i n}$. Of course, by swapping the input and output terminals, the conventional converter can generate steppedup and/or stepped-down voltages. However, the conversion ratios of the conventional multi-output SC converter are predetermined by the circuit topology. For this reason, the application filed of the conventional converter is limited.

\subsection{Proposed Converter}

Figure 2 shows the proposed SC SIDO converter. The proposed SIDO converter provides stepped-up and/or 
stepped-down voltages as follows:

$$
V_{\text {out } 1}=\left(\frac{s_{1}}{r}\right) V_{\text {in }} \quad \text { and } \quad V_{\text {out } 2}=\left(\frac{s_{2}}{r}\right) V_{\text {in }},
$$

where $\quad s_{1} \neq s_{2} \quad\left(r, s_{1}, s_{2}=\{1,2, \ldots, N\}\right)$.

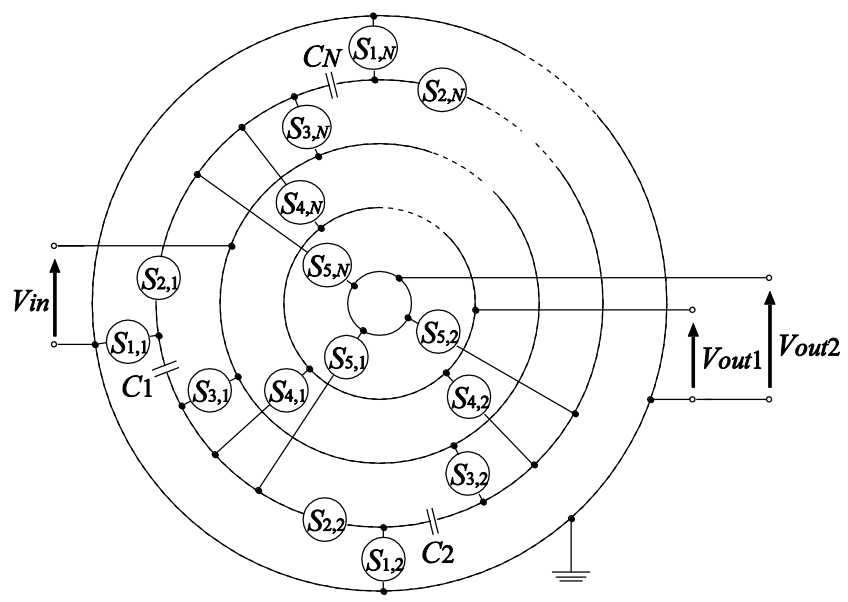

(a) General form.

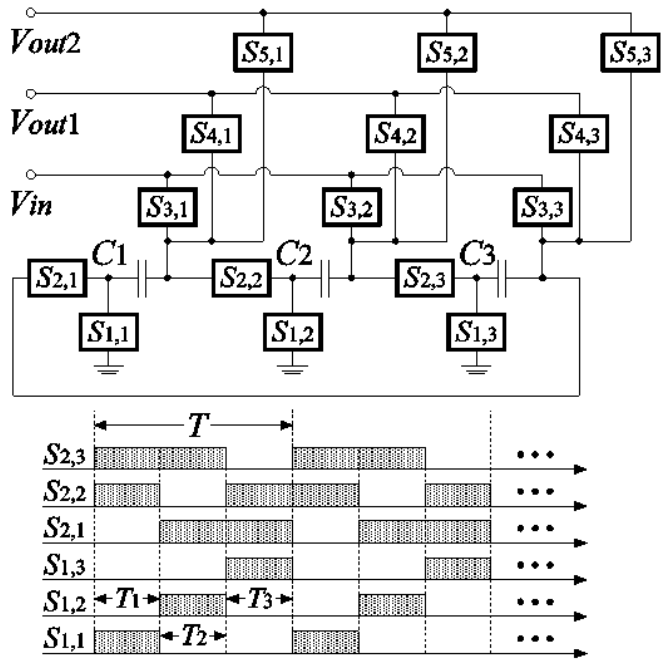

(b) Three stages $(N=3)$.

Fig. 2. Proposed SC SIDO converter.

For easy understanding, let's discuss the simplest example of the proposed SIDO converter. Figure 2 shows an example of the proposed SIDO converter. In figure 2 (b), clock pulses for the transistor switch $S_{1, j}(j=1,2,3)$ are non-overlapped three phase pulses and clock pulses for $S_{2, j}$ are set to inverted pulses of $S_{1, j}$. According to the conversion ratios $s_{1} / r$ and $s_{2} / r$ in (2), the transistor switches $S_{3, j}, S_{4, j}$, and $S_{5, j}$ are driven by clock pulses obtained by shifting the clock pulse of $S_{1, j}$ cyclically. In other words, by controlling $S_{3, j}, S_{4, j}$, and $S_{5, j}$, the conversion ratios $s_{1} / r$ and $s_{2} / r$ are determined. Concretely, the proposed SIDO converter of figure 2 (b) can offer the output voltages, $V_{\text {out } 1}$, $V_{\text {out } 2}=\left\{V_{\text {in }} / 3, V_{\text {in }} / 2,2 V_{\text {in }} / 3, V_{\text {in }}, 3 V_{\text {in }} / 2,2 V_{\text {in }}, 3 V_{\text {in }}\right\}$, without changing circuit topology.

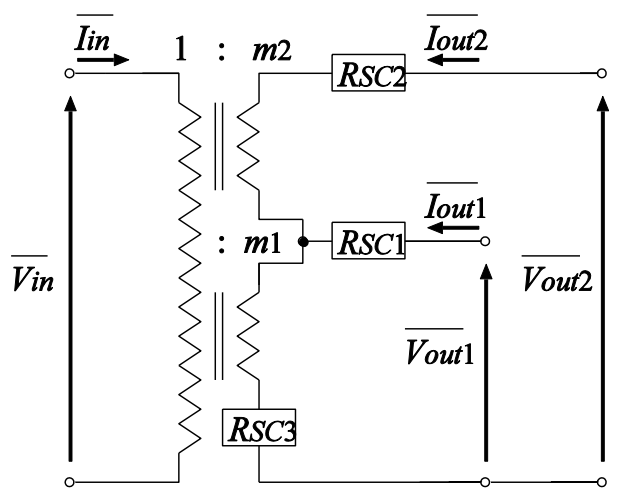

Fig. 3. Proposed equivalent circuit.

\section{Theoretical Analysis}

To save space, the property of the proposed SIDO converter is analyzed in conversion ratios of $1 / 2$ and $3 / 2$ theoretically. By assuming a five-terminal equivalent circuit as shown in figure 3 , the proposed analysis method is performed, because it is known that the general equivalent circuit of the single-input single-output SC DC-DC converter can be expressed by a four-terminal circuit ${ }^{(14)}$. In figure $3, R_{s c 1}, R_{s c 2}$, and $R_{s c 3}$ are called the $\mathrm{SC}$ resistance and $m_{1}$ and $m_{2}$ are the conversion ratio of ideal transformers. Unlike the state-space averaging $\operatorname{method}^{(12,13)}$, the proposed analysis method derives these parameters from instantaneous equivalent circuits of the SC SIDO converter without complex matrix calculations.

Figure 4 shows the instantaneous equivalent circuits of the proposed SIDO converter of figure 2 (b). In figure 4 , $R_{\text {on }}$ denotes the on-resistance of the transistor switch. In the steady state, the differential value of electric charges in $C_{k}$ $(k=1,2,3)$ satisfies the following equations:

$$
\sum_{i=1}^{3} \Delta q_{T_{i}}^{k}=0,
$$

where $\Delta q_{T i}{ }^{k}((i=1,2,3)$ and $(k=1,2,3))$ denotes the electric charge of the $k$-th capacitor in the case of State- $T_{i}$. The interval of State- $T_{i}$ satisfies the following conditions:

$$
T=\sum_{i=1}^{3} T_{i} \quad \text { and } \quad T_{1}=T_{2}=T_{3},
$$

where $T$ is a period of the clock pulse and $T_{i}(i=1,2,3)$ is 
the interval of State- $T_{i}$. In State- $T_{1}$, the differential values of electric charges in the input $V_{\text {in }}$ and the outputs $V_{\text {out } 1}$ and $V_{\text {out } 2}, \Delta q_{T 1, \text { Vin }}, \Delta q_{T 1, \text { Vout } 1}$ and $\Delta q_{T 1, \text { Vout } 2}$, are expressed as

$$
\begin{aligned}
& \Delta q_{T_{1}, V_{\text {in }}}=\Delta q_{T_{1}}^{2}-\Delta q_{T_{1}}^{3}, \\
& \Delta q_{T_{1}, V_{\text {out } 1}}=\Delta q_{T_{1}}^{1}-\Delta q_{T_{1}}^{2},
\end{aligned}
$$$$
\text { and } \quad \Delta q_{T_{1}, V_{\text {out } 2}}=\Delta q_{T_{1}}^{3} \text {. }
$$

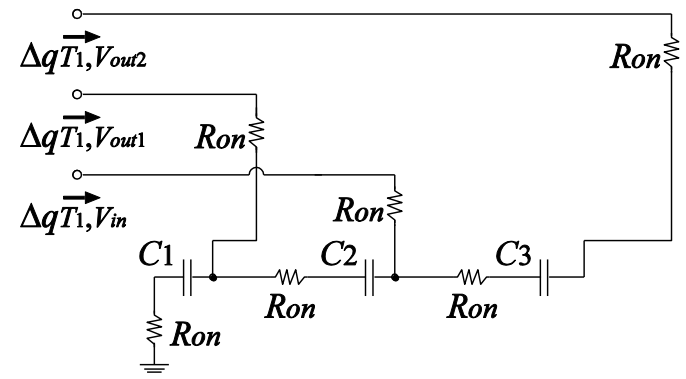

(a) State- $T_{1}$.

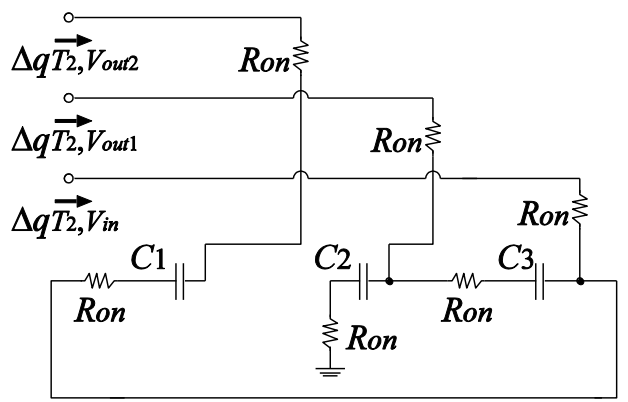

(b) State- $T_{2}$.

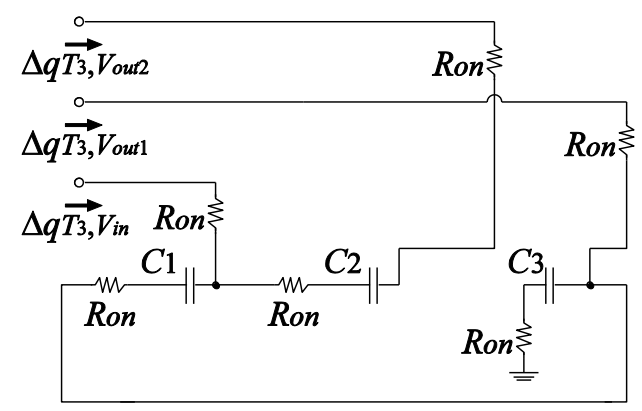

(c) State- $T_{3}$.

Fig. 4. Instantaneous equivalent circuits with conversion ratios of $1 / 2$ and $3 / 2$.

In State- $T_{2}$, the differential values of electric charges in the input $V_{\text {in }}$ and the outputs $V_{\text {out } 1}$ and $V_{\text {out } 2}, \Delta q_{T 2, \text { Vin }}$, $\Delta q_{T 2, \text { Vout } 1}$ and $\Delta q_{T 2, \text { Vout } 2}$, are expressed as

$$
\begin{aligned}
& \Delta q_{T_{2}, V_{\text {in }}}=\Delta q_{T_{2}}^{3}-\Delta q_{T_{2}}^{1}, \\
& \Delta q_{T_{2}, V_{\text {out } 1}}=\Delta q_{T_{2}}^{2}-\Delta q_{T_{2}}^{3}, \\
& \text { and } \quad \Delta q_{T_{2}, V_{\text {out } 2}}=\Delta q_{T_{2}}^{1} .
\end{aligned}
$$

In State- $T_{3}, \Delta q_{T 3, \text { Vin }}, \Delta q_{T 3, \text { Vout } 1}$ and $\Delta q_{T 3 \text {,Vout } 2}$, are expressed as

$$
\begin{aligned}
& \Delta q_{T_{3}, V_{\text {in }}}=\Delta q_{T_{3}}^{1}-\Delta q_{T_{3}}^{2}, \\
& \Delta q_{T_{3}, V_{\text {out } 1}}=\Delta q_{T_{3}}^{3}-\Delta q_{T_{3}}^{1}, \\
& \Delta q_{T_{3}, V_{\text {out } 2}}=\Delta q_{T_{3}}^{2} .
\end{aligned}
$$$$
\text { and }
$$

Furthermore, the following conditions are satisfied, because the instantaneous equivalent circuits have symmetrical structure as shown in figure 4.

$$
\begin{aligned}
& \Delta q_{T_{1}, V_{\text {in }}}=\Delta q_{T_{2}, V_{\text {in }}}=\Delta q_{T_{3}, V_{\text {in }}}, \\
& \Delta q_{T_{1}, V_{\text {out } 1}}=\Delta q_{T_{2}, V_{\text {out } 1}}=\Delta q_{T_{3}, V_{\text {out } 1}}, \\
& \Delta q_{T_{1}, V_{\text {out } 2}}=\Delta q_{T_{2}, V_{\text {out } 2}}=\Delta q_{T_{3}, V_{\text {out }} 2}, \\
& \Delta q_{T_{1}}^{2}=\Delta q_{T_{2}}^{3}=\Delta q_{T_{3}}^{1}, \\
& \Delta q_{T_{1}}^{3}=\Delta q_{T_{2}}^{1}=\Delta q_{T_{3}}^{2}, \\
& \text { and } \Delta q_{T_{1}}^{1}=\Delta q_{T_{2}}^{2}=\Delta q_{T_{3}}^{3} .
\end{aligned}
$$

Using (5)-(7), the average input current and the average output currents can be expressed as

$$
\begin{array}{ll} 
& \overline{I_{\text {in }}}=\frac{\Delta q_{V_{\text {in }}}}{T}=\sum_{i=1}^{3} \frac{\Delta q_{T_{i}, V_{\text {in }}}}{T}, \\
\overline{I_{\text {out } 1}} & =\frac{\Delta q_{V_{\text {out } 1}}}{T}=\sum_{i=1}^{3} \frac{\Delta q_{T_{i}, V_{\text {out } 1}}}{T}, \\
\text { and } \quad \overline{I_{\text {out } 2}} & =\frac{\Delta q_{V_{\text {out } 2}}}{T}=\sum_{i=1}^{3} \frac{\Delta q_{T_{i}, V_{\text {out } 2}}}{T} .
\end{array}
$$

In (9), $\Delta q_{V i n}, \Delta q_{\text {Vout } 1}$, and $\Delta q_{\text {Vout } 2}$ are electric charges in $V_{\text {in }}$, $V_{\text {out } 1}$, and $V_{\text {out } 2}$, respectively. Substituting (3)-(8) into (9), we have the relation between the average input current and the average output currents as follows:

$$
\overline{I_{\text {in }}}=-\frac{1}{2} \overline{I_{\text {out } 1}}-\frac{3}{2} \overline{I_{\text {out } 2}} \text {. }
$$

From (10), the conversion ratios in figure 3 are obtained as $m_{1}=1 / 2$ and $m_{2}=3 / 2$.

Next, in order to derive the SC resistances $R_{S C 1}, R_{S C 2}$, 
and $R_{S C 3}$, the consumed energy in one period is discussed. From figure 4 (a), the consumed energy $W_{T}$ can be expressed as

$$
\begin{aligned}
& \qquad \begin{array}{l}
W_{T}=\sum_{i=1}^{3} W_{T_{i}}=3 W_{T_{1}}, \\
\text { where } \quad W_{T_{1}}=\frac{\left(\Delta q_{T_{1}}^{1}\right)^{2}}{T_{1}} R_{\text {on }}+\frac{\left(\Delta q_{T_{1}}^{2}\right)^{2}}{T_{1}} R_{\text {on }} \\
+\frac{2\left(\Delta q_{T_{1}}^{3}\right)^{2}}{T_{1}} R_{\text {on }}+\frac{\left(\Delta q_{T_{1}, V_{\text {out } 1}}\right)^{2}}{T_{1}} R_{\text {on }} \\
+\frac{\left(\Delta q_{T_{1}, V_{\text {in }}}\right)^{2}}{T_{1}} R_{\text {on }} .
\end{array}
\end{aligned}
$$

Here, the consumed energy $W_{T}$ of the five-terminal equivalent circuit shown in figure 3 is obtained as

$$
\begin{aligned}
W_{T}= & R_{S C 1}\left(\overline{I_{\text {out } 1}}\right)^{2} T+R_{S C 2}\left(\overline{I_{\text {out } 2}}\right)^{2} T \\
& +R_{S C 3}\left(\overline{I_{\text {out } 1}}+\overline{I_{\text {out } 2}}\right)^{2} T \\
= & \left(R_{S C 1}+R_{S C 3}\right) \frac{\left(\Delta q_{V_{\text {out } 1}}\right)^{2}}{T} \\
& +\left(R_{S C 2}+R_{S C 3}\right) \frac{\left(\Delta q_{V_{\text {out } 2}}\right)^{2}}{T} \\
& +2 R_{S C 3} \frac{\left(\Delta q_{V_{\text {out } 1}} \cdot \Delta q_{V_{\text {out } 2}}\right)}{T} .
\end{aligned}
$$

Therefore, from (12) and (13), we have the SC resistances as follows:

$$
R_{S C 1}=R_{o n}, \quad R_{S C 2}=4 R_{o n}, \quad \text { and } R_{S C 3}=\frac{3}{4} R_{o n} .
$$

By combining (10) and (14), the equivalent circuit of the proposed SIDO converter can be expressed by figure 5 , where $R_{L 1}$ and $R_{L 2}$ are output loads.

From figure 5, the power efficiency and the output voltages of the proposed SIDO converter can be derived. By using a principle of superposition, we have the output voltages $V_{\text {out } 1}$ and $V_{\text {out } 2}$ as follows:

$$
V_{\text {out } 1}=V_{\text {out } 1,1}+V_{\text {out } 2,1} \text {, }
$$

where $\quad V_{\text {out } 1,1}=\frac{\left(V_{\text {in }} / 2-V_{x 1}\right) R_{L 1}}{R_{S C 1}+R_{L 1}}$,

$$
V_{\text {out } 2,1}=\frac{\left(V_{\text {in }} / 2-V_{x 1}\right) R_{L 2}}{R_{S C 2}+R_{L 2}},
$$

$$
\text { and } \quad V_{x 1}=\frac{\left(V_{i n} / 2\right) R_{S C 3}}{R_{S C 3}+\left(R_{S C 1}+R_{L 1}\right) / /\left(R_{S C 2}+R_{L 2}\right)}
$$

and

$$
V_{\text {out } 2}=V_{\text {out } 1,2}+V_{\text {out } 2,2}
$$

where

$$
V_{\text {out } 1,2}=\frac{-V_{x 2} R_{L 1}}{R_{S C 1}+R_{L 1}}
$$

$$
\begin{gathered}
V_{\text {out } 2,2}=\frac{\left(V_{\text {in }}-V_{x 2}\right) R_{L 2}}{R_{S C 2}+R_{L 2}}, \\
\text { and } \quad V_{x 2}=\frac{\left\{\left(R_{S C 1}+R_{L 1}\right) / / R_{S C 3}\right\} V_{i n}}{\left(R_{S C 2}+R_{L 2}\right)+\left(R_{S C 1}+R_{L 1}\right) / / R_{S C 3}} .
\end{gathered}
$$

Furthermore, the power efficiency $\eta$ of the proposed SIDO converter is obtained as

$$
\begin{aligned}
& \eta=\frac{R_{L 1}+R_{L 2} \alpha^{2}}{\left(R_{S C 1}+R_{L 1}\right)+\left(R_{S C 2}+R_{L 2}\right) \alpha^{2}+R_{S C 3}(1+\alpha)^{2}}, \\
& \text { where } \quad \alpha=\frac{3\left(R_{S C 1}+R_{L 1}\right)+2 R_{S C 3}}{R_{S C 2}-2 R_{S C 3}+R_{L 2}} .
\end{aligned}
$$

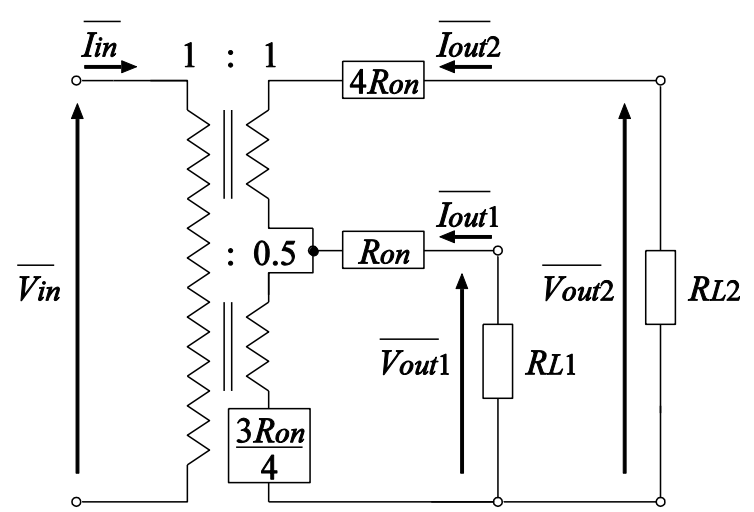

Fig. 5. Equivalent circuit of the SC SIDO converter with conversion ratios of $1 / 2$ and $3 / 2$.

\section{Simulations}

To confirm the validity of theoretical analysis, 
properties of the proposed SIDO converter are investigated by SPICE simulations. The SPICE simulations are performed under conditions that $V_{\text {in }}=3.7 \mathrm{~V}, C_{1}=C_{2}=C_{3}=$ $C_{\text {out } 1}=C_{\text {out } 2}=1 \mu \mathrm{F}, R_{\text {on }}=1 \Omega, T=1.5 \mu \mathrm{s}$ and $R_{L}=R_{L 1}=R_{L 2}$, where $C_{\text {out } 1}$ and $C_{\text {out } 2}$ are output capacitors connecting the output terminals. In the SPICE simulations, the property of the proposed converter is compared with that of the conventional serial fix type converter shown in figure 1.

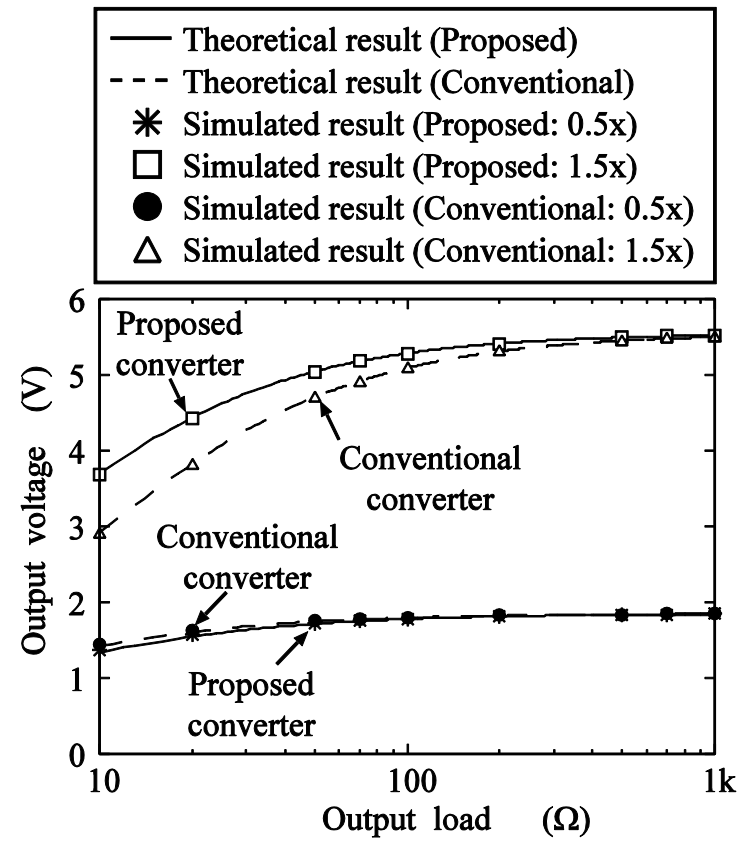

(a) Output voltages as a function of the output load.

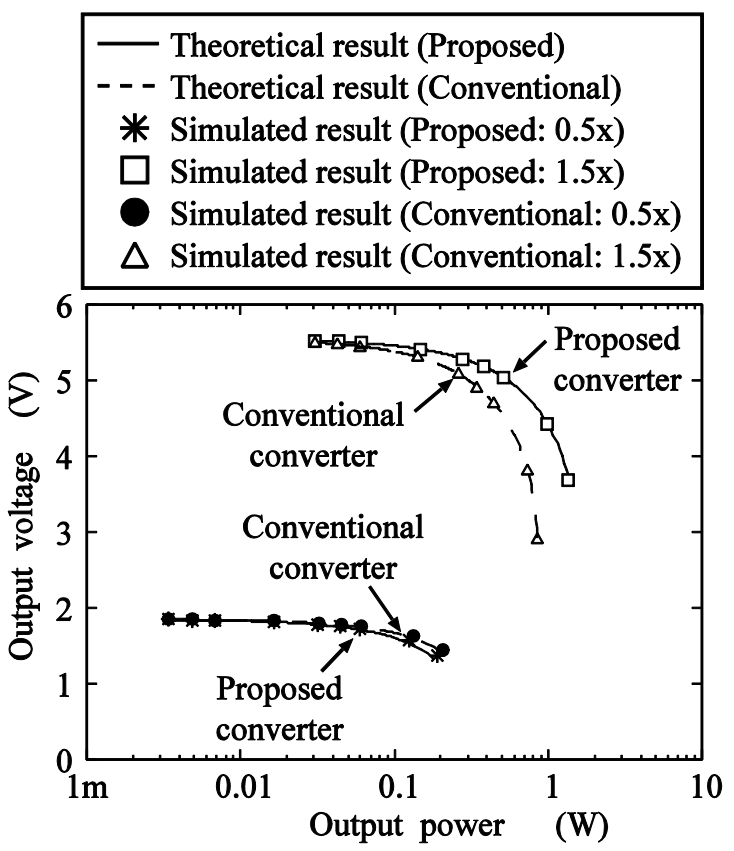

(b) Output voltages as a function of the output power.

Fig. 6. Output voltages of the proposed SIDO converter.
Table 1. Timing of clock pulses for conversion ratios of $1 / 2$ and $3 / 2$.

\begin{tabular}{|c|c|c|}
\hline State & On & Off \\
\hline$T_{1}$ & $S_{1,1}, S_{2,2}, S_{2,3}, S_{3,2}, S_{4,1}, S_{5,3}$ & Others \\
\hline$T_{2}$ & $S_{1,2}, S_{2,3}, S_{2,1}, S_{3,3}, S_{4,2}, S_{5,1}$ & Others \\
\hline$T_{3}$ & $S_{1,3}, S_{2,1}, S_{2,2}, S_{3,1}, S_{4,3}, S_{5,2}$ & Others \\
\hline
\end{tabular}

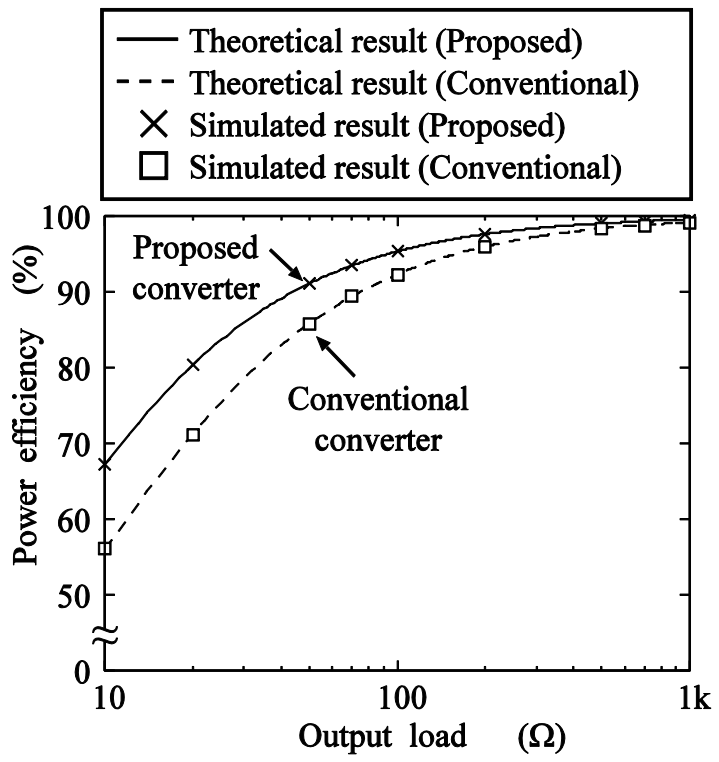

(a) Power efficiency as a function of the output load.

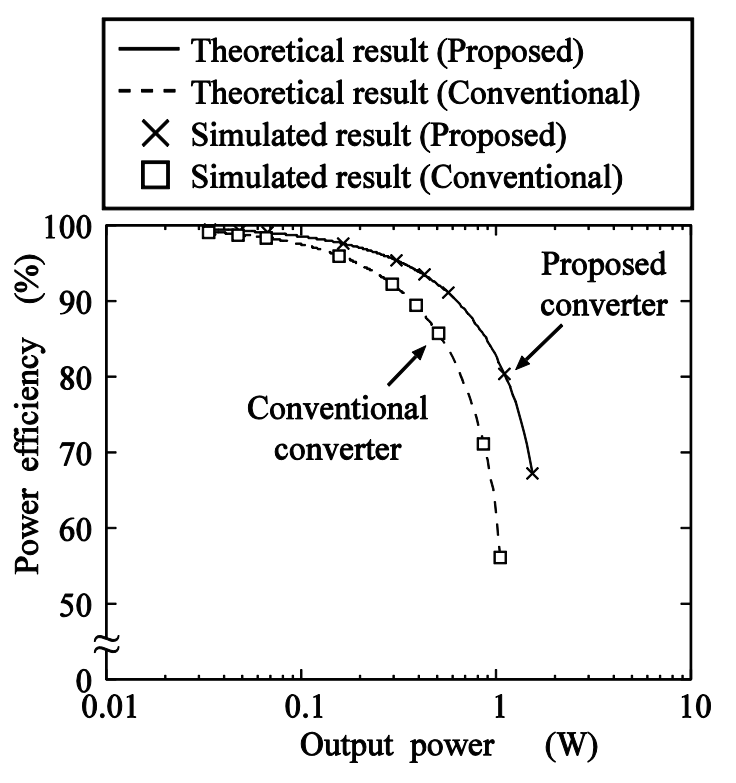

(b) Power efficiency as a function of the output power.

Fig. 7. Power efficiency of the proposed SIDO converter.

Figure 6 shows the simulated output voltages with conversion ratios of $1 / 2$ and $3 / 2$. In figure 6 , the clock pulse of the proposed converter was set to as shown in Table 1 . On the other hand, in the conventional converter of figure 1 , 
the input terminal $V_{\text {in }}$ and the output terminals $V_{\text {out } 1}$ and $V_{\text {out } 2}$ are set to the terminal $b, a$, and $c$, respectively. In figures 6 (a) and (b), the solid lines show the theoretical results obtained by (15) and (16). As figure 6 shows, the theoretical results are in good agreement with the SPICE simulated results. Therefore, the validity of (15) and (16) can be confirmed. Furthermore, unlike the conventional SIDO converter shown in figure 1, the proposed SIDO converter can provide not only a stepped-down voltage but also a stepped-up voltage without changing circuit topology.

Figure 7 shows the simulated power efficiency with conversion ratios of $1 / 2$ and $3 / 2$. In figures 7 (a) and (b), the solid lines show the theoretical results obtained by (17). As figure 7 shows, theoretical results agree well with SPICE simulated results. From figures 6 and 7, the validity of the theoretical analysis can be confirmed. The derived theoretical equations will be helpful to estimate the properties of the proposed SC SIDO converter. Furthermore, as figure 7 shows, the proposed converter can improve the power efficiency more than $3 \%$ from the conventional converter when the output load is $100 \Omega$. The proposed converter can achieve more than $82 \%$ efficiency over a range of output power from $0.1 \mathrm{~W}$ to $1 \mathrm{~W}$.

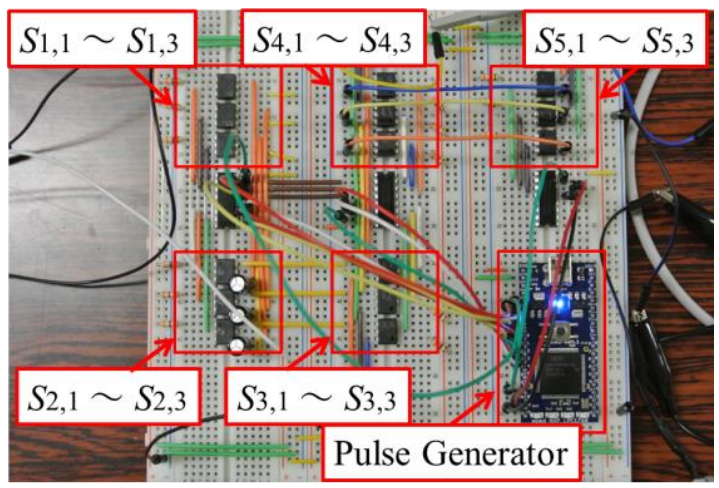

Fig. 8. Experimental circuit.

\section{Experiment}

Figure 8 shows the photograph of the experimental circuit. The experimental circuit of figure 8 was built with commercially available ICs AQV212 and TD62004APG on a bread board, where $V_{\text {in }}=3.7 \mathrm{~V}, C_{1}=C_{2}=C_{3}=C_{\text {out } 1}=C_{\text {out } 2}$ $=10 \mu \mathrm{F}, T=1.5 \mathrm{~ms}$ and $R_{L 1}=R_{L 2}=10 \mathrm{k} \Omega$. In the experiments, we focused on the verification of the circuit topology, because the experimental circuit was built with commercially available ICs on a bread board.
Figure 9 shows the measured output voltages of the proposed SC SIDO converter, where the $1 / 2 \mathrm{x}$ and $3 / 2 \mathrm{x}$ conversion ratios are realized. In figure 9 , the measured output voltages are $1.84 \mathrm{~V}$ and $5.53 \mathrm{~V}$, where the ideal outputs are $1.85 \mathrm{~V}$ and 5.55 . Figure 10 shows the measured output voltages with conversion ratios of $1 / 3$ and $2 / 3$. In figure 10 , the measured output voltages are $1.23 \mathrm{~V}$ and $2.46 \mathrm{~V}$, where the ideal outputs are $1.23 \mathrm{~V}$ and 2.47 . As figures 9 and 10 shows, the proposed converter can control conversion ratios without changing circuit topology. From figures 9 and 10, the validity of the proposed SC SIDO topology can be confirmed.

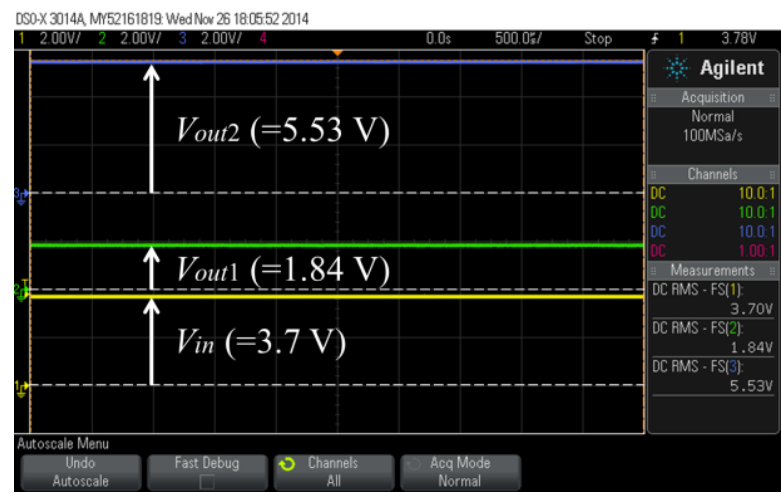

Fig. 9. Measured outputs with conversion ratios of $1 / 2$ and $3 / 2$.

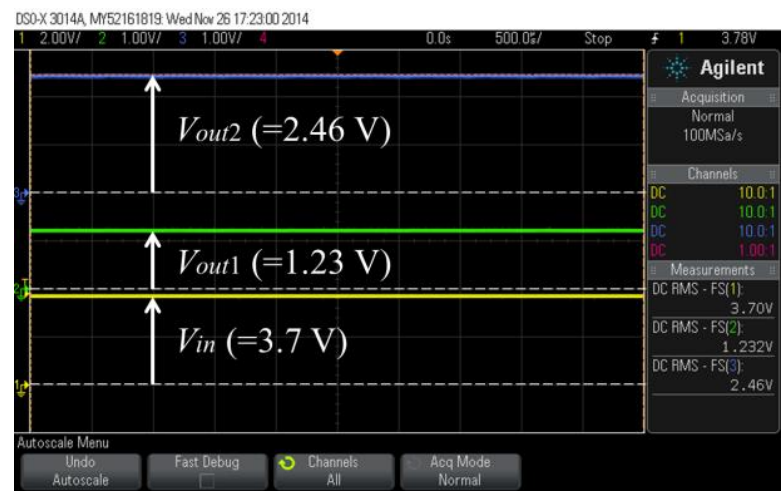

Fig. 10. Measured outputs with conversion ratios of $1 / 3$ and $2 / 3$.

\section{Conclusions}

An SC SIDO DC-DC converter and its analysis method have been proposed in this paper. Concerning the proposed converter, SPICE simulations, theoretical analysis, and experiments were performed to confirm the validity of the circuit design. 
The results of this study are as follows: (1) Without changing circuit topology, not only a stepped-down voltage but also a stepped-up voltage was offered by the proposed SIDO converter; (2) the proposed converter improved power efficiency more than $3 \%$ from the serial fix type converter when the output load was $100 \Omega$. Furthermore, more than $82 \%$ efficiency was obtained over a range of output power from $0.1 \mathrm{~W}$ to $1 \mathrm{~W}$ with conversion ratios of $1 / 2$ and $3 / 2$; and (3) handy theoretical equations to estimate properties of the proposed SIDO converter were obtained by using a five-terminal equivalent circuit. The theoretical results were in good agreement with SPICE simulated results.

The detailed experiment of the proposed converter is left to a future study.

\section{Acknowledgment}

This work was supported by JSPS KAKENHI Grant Number 24531193.

\section{References}

(1) Pradipta Patra, Amit Patra and Debaprasad Kastha : "On-chip implementation of a multi-output voltage regulator based on single inductor buck converter topology", 20th International Conference on VLSI Design, pp. 935-940, 2007

(2) Jaya Deepti Dasika, Behrooz Bahrani, Maryam Saeedifard, Alireza Karimi, and Alfred Rufer : "Multivariable control of single-inductor dual-output buck converters", IEEE Trans. on Power Electronics, Vol. 29, No. 4, pp. 2061-2070, 2014

(3) Olive Ray, Anil Prasad Josyula, and Santanu Mishra : "A multi-port DC-DC converter topology with simultaneous buck and boost outputs", IEEE International Symposium on Industrial Electronics (ISIE), pp.1-6, 2013

(4) Olive Ray, Anil Prasad Josyula, Santanu Mishra, and Avinash Joshi : "Integrated dual-output converter", IEEE Trans. on Industrial Electronics, 2014 (IEEE Early Access Articles)

(5) Stefan Dietrich, Sebastian Strache, Lukas Lohaus, Ralf Wunderlich, and Stefan Heinen : "A capacitor-free single-inductor multiple-output LED driver", 39th Annual Conference of the IEEE Industrial Electronics Society, pp. 6034-6039, 2013

(6) Shoji Suzuki, Ichirou Oota, Noriaki Hara, and Fumio
Ueno : "A new serial fix type switched-capacitor DC-DC converter with a low ripple input-current", 29th Annual IEEE Power Electronics Specialists Conference, pp.1517-1522, 1998

(7) Kei Eguchi, Ichirou Oota, Shinya Terada, and Hongbing Zhu : "Synthesis and analysis of a switched-capacitor-based battery equalizer using levelshift circuits", International Journal of Intelligent Engineering and Systems, Vol.5, No.4, pp.1-9, 2012

(8) Zhe Hua, Hoi Lee, and Xiwen Zhang : "An auto-reconfigurable dual-output SC DC-DC regulator with sub-harmonic fixed on-time control for energyharvesting applications", IEEE International Symposium on Circuits and Systems (ISCAS), pp.1472-1475, 2013

(9) Kei Eguchi, Kuniaki Fujimoto, and Hirofumi Sasaki : "A hybrid input charge-pump using micropower thermoelectric generators", IEEJ Trans. on Electrical and Electronic Engineering, Vol.7, No.4, pp.415-422, 2012

(10) Chia-Min Chen, Chun-Yen Chiang, Chen-Cheng Du, Fang-Ting Chou, and Chung-Chih Hung : "Dual-output switched-capacitor DC-DC converter with peseudothree-phase swap-and-cross control and amplitude modulation mechanism", IEEE Asian Solid-State Circuits Conference, pp.57-60, 2013

(11) Yuval Beck and Sigmond Singer : "Capacitive transposed series-parallel topology with fine tuning capabilities", IEEE Trans. on Circuits and Systems I, Vol.58, Issue 1, pp.51-61, 2011

(12) Madan M. Jalla, Ali Emadi, Geoffrey A. Williamson, and Babak Fahimi : "Real time state estimation of multi-converter more electric ship power systems using the generalized state space averaging method", 30th Annual Conference of IEEE Industrial Electronics Society, Vol.2, pp. 1514-1519, 2004

(13) Sihun Yang, Kenta Goto, Yasutaka Imamura, and Masahito Shoyama : "Dynamic characteristics model of bi-directional DC-DC converter using state-space averaging method", IEEE 34th International Telecommunications Energy Conference, pp.1-5, 2012

(14) Kei Eguchi, Prasit Julsereewong, Amphawan Julsereewong, Kuniaki Fujimoto, and Hirofumi Sasaki : "A Dickson-type adder/subtractor DC-DC converter realizing step-up/step-down conversion", International Journal of Innovative Computing, Information and Control, Vol.9, No.1, pp.123-138, 2013 